\title{
Planificación preoperatoria de los colgajos de perforantes
}

\section{Preoperative planning on perforator flaps}

\author{
Masià, J .*, Clavero, J . A.**, Carrera, A.***
}

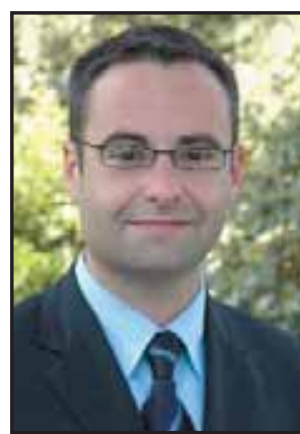

Masià, J.
Resumen

La valoración preoperatoria precisa de la anatomía vascular es altamente valiosa para conseguir la mejor planificación quirúrgica y poder realizar colgajos de perforantes de una forma más rápida y segura. La localización de la mejor perforante y la valoración de su trayecto hasta el vaso origen es esencial para definir la perforante ideal para nuestro colgajo. Por tanto, después de estudiar el tipo de defecto a reconstruir, el punto clave será el encontrar una perforante con un flujo sanguíneo adecuado, una localización apropiada y una disección sencilla.
Palabras clave Colgajos perforantes, Planificación preoperatoria

Código numérico 158336
Key words

Perforator flaps, Preoperative evaluation

Código numérico 158336

* Director del Servicio de Cirugía Plástica y Reparadora, Hospital de la Santa Creu i Sant Pau. Universidad Autònoma de Barcelona, Barcelona. España. 


\section{Introducción}

En cualquier procedimiento quirúrgico es fundamental una adecuada planificación preoperatoria para poder conseguir con éxito el resultado final. Tras una correcta valoración del objetivo o defecto a reconstruir, del estado general del paciente y de las posibles zonas donantes disponibles, en la cirugía de perforantes deberíamos intentar localizar la perforante adecuada para elevar nuestro colgajo.

En 1987, Ian Taylor, con su trabajo sobre los territorios cutáneos y los angiosomas (1), puso la primera piedra para el desarrollo de los colgajos de perforantes. A los 10 años de publicados sus estudios se consolidaban algunas de las aplicaciones clínicas de estos colgajos; cirujanos como Koshima (2), Allen (3), Wei (4) y Blondeel (5) han sido los verdaderos promotores de este tipo de cirugía. En la actualidad, no podemos prescindir en nuestro "armamentarium" reconstructivo de los colgajos de perforantes; si queremos ofrecer una Cirugía Plástica propia del siglo XXI debemos entender y poder aplicar este tipo de colgajos.

El conocimiento adecuado de la vascularización y perfusión tisular de los territorios cutáneos junto a un mejor dominio de las técnicas de disección microquirúrgica proporcionan la posibilidad de realizar reconstrucciones cada vez más apropiadas para solucionar los problemas de nuestros pacientes. De esta manera basándonos en los principios básicos de la especificidad reconstructiva y de minimización de la morbilidad, podemos elegir los colgajos óptimos para cada defecto y mejorar nuestros resultados.

Desafortunadamente, a pesar de los avances en el conocimiento anatómico de los territorios cutáneos, no es posible realizar un mapeo sistemático de la localización precisa de las perforantes dominantes (6). La enorme variabilidad anatómica de las perforantes a nivel inter-individual e intra-individual, hace que el disponer de un sistema fiable de localización preoperatoria de los vasos en que vamos a basar la transferencia de nuestros colgajos sea de una enorme utilidad (Fig. 1).

Por tanto podemos definir como puntos clave para la correcta ejecución de los colgajos de perforantes los siguientes:

- Definir, de la forma más precisa posible, la anatomía de nuestra perforante (localización, trayecto intramuscular, ramificación intratisular, etc).

- Realizar el mapeo de las perforantes en la misma posición del paciente sobre la mesa operatoria.

- Valorar posibles alteraciones anatómicas provocadas por cirugías previas o secuelas cicatriciales, así como variaciones anatómicas de la normalidad.

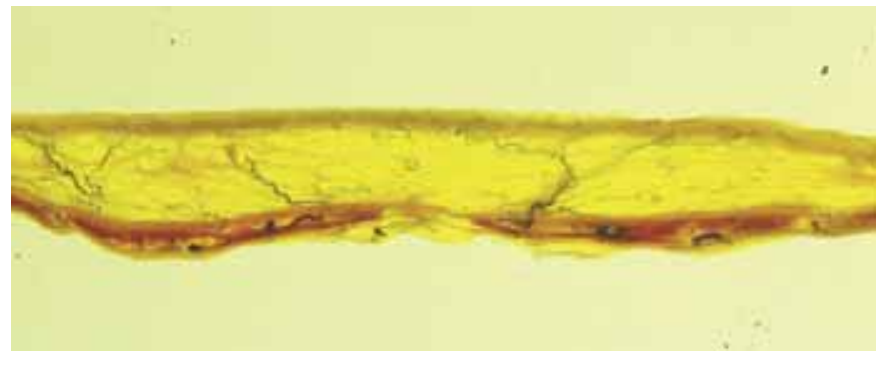

Fig. 1. Transparentación de una pieza anatómica abdominal (sección transversal), donde se observan diferentes perforantes periumbilicales con su ramificación intratisular.

- Preservar otras alternativas vasculares para poder afrontar complicaciones, como venas superficiales u otras perforantes, hasta el final de la cirugía.

La selección de la perforante más adecuada la haremos en función de los siguientes criterios que nos ayudarán a elegir la perforante ideal para cada colgajo:

- Calibre y pulsabilidad de la perforante. Es importante poder analizar estos aspectos antes de iniciar la disección, ya que a veces la manipulación de la misma puede ocasionar vasoespasmo y dificultar una correcta valoración (Fig. 2).

- Adecuada localización de la perforante en el contexto del tejido a transferir. Es recomendable que esté lo más centrada posible para poder perfundir correctamente todo el tejido.

- Elegiremos la perforante con el menor trayecto intramuscular posible, para lesionar lo menos posible el músculo con nuestra manipulación y para que la disección sea lo menos tediosa posible.

Una vez que sabemos lo que necesitamos para nuestro colgajo, debemos conocer los diferentes métodos para realizar el estudio o mapeo de las perforantes; actualmente disponemos del Doppler de

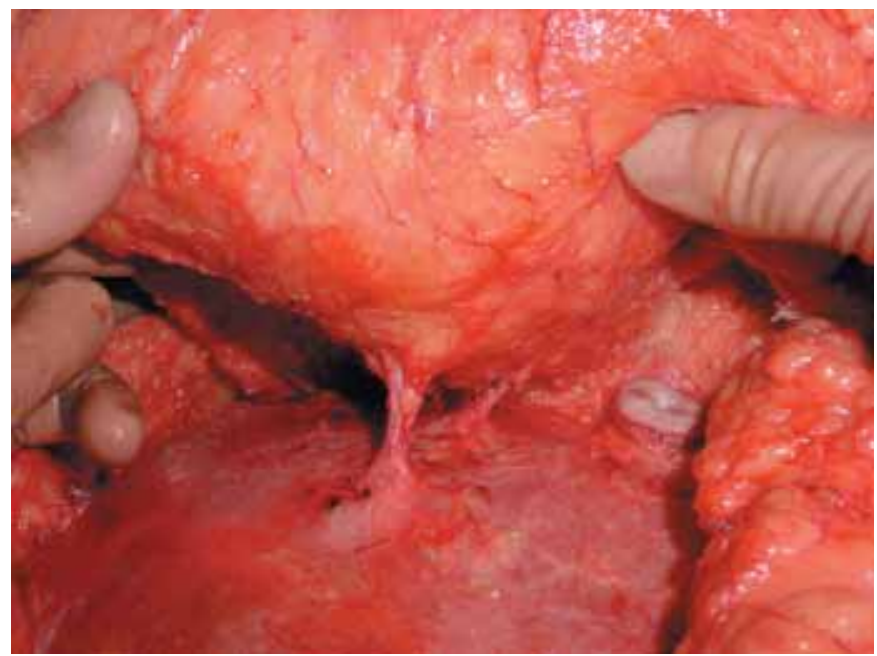

Fig. 2. Disección suprafascial y valoración de una perforante de un DIEP. 


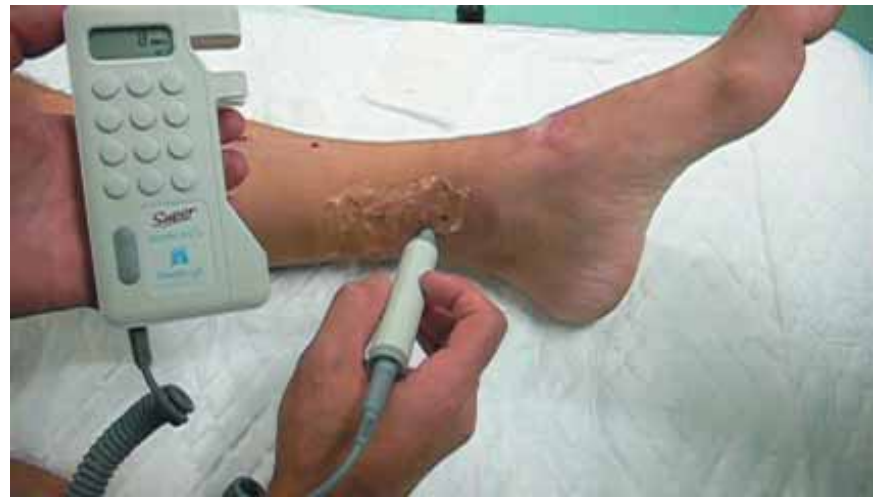

Fig. 3. Mapeo de las perforantes de la tibial posterior mediante un Doppler de ultrasonidos.

ultrasonido, el Doppler duplex color y del escáner de multidetectores (6-9).

\section{DOPPLER DE ULTRASONIDO}

El Doppler acústico unidireccional es ampliamente conocido. Su facilidad de uso y su disponibilidad al poderlo encontrar en cualquier medio hospitalario, hacen que sea el sistema más universal en el estudio de perforantes. Su portabilidad, bajo coste y relativa sencillez en la interpretación de las señales acústicas, han hecho que se incorporara a la practica de la microcirugía desde sus inicios en los años 70 (Fig. 3).

Las sondas más utilizadas son las de $8 \mathrm{mHz}$, aunque hay quién prefiere las de $5 \mathrm{mHz}$. Con ellas podemos detectar el flujo de los vasos más superficiales, pudiendo diferenciar entre flujos arteriales y venosos. Es un método de alta sensibilidad pero de baja especificidad, ya que solo permite una valoración subjetiva de los flujos mediante el sonido $(6,7)$. Esto hace que a menudo la clasificación de las perforantes según la dominancia sonora no corresponda con el tamaño real, y vasos con una pulsabilidad sonora aceptable, no sean lo suficiente vigorosos como para perfundir todo un colgajo. De la misma manera, pequeños vasos axiales pueden ser confundidos con perforantes, produciendo un número no despreciable de falsos positivos (6).

Por todos estos motivos, a nuestro parecer, el Doppler de ultrasonidos es un método que debido a su sencillez y a pesar de sus inconvenientes, puede servir para una primera aproximación en la localización de las perforantes, es decir, únicamente como orientación. Por tanto siempre que no dispongamos de otros medios más fiables deberemos esperar a la confirmación intraoperatoria para realizar el diseño final de nuestro colgajo.

\section{COLOR DUPLEX DOPPLER}

Durante la última década, los avances tecnológicos en este tipo de Doppler han permitido escanear vasos

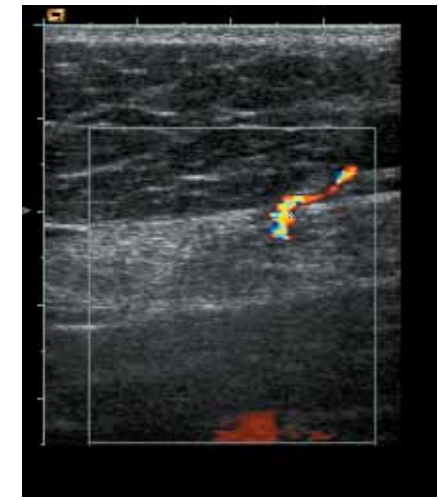

Fig. 4. Imagen de una perforante abdominal mediante Color Duplex Doppler.

de diámetro de hasta $0.2 \mathrm{~mm}$ desde la superficie cutánea hasta los planos más profundos. Por tanto podemos afirmar que la información que podemos obtener con este método es totalmente fiable.

$\mathrm{Su}$ alta sensibilidad permite una detallada evaluación del calibre, flujo y relaciones anatómicas de las perforantes (Fig. 4). A pesar de su valor predictivo del $100 \%$, ha tenido una escasa implantación en los centros debido a que el tiempo por prueba y paciente es muy largo. Requiere casi una hora por exploración y mantener la misma posición durante todo el estudio, lo que lo hace inviable para la mayoría de sistemas sanitarios y hospitales. Aparte de la incomodidad que significan este tipo de pruebas para los pacientes, se requiere personal muy especializado para realmente poder estudiar y localizar los vasos con fiabilidad, siendo su valoración muy técnico-dependiente y conllevando por tanto una baja especificidad. Otro de los inconvenientes que presenta es su dificultad de interpretación por parte de los cirujanos plásticos, ya que al obtener imágenes ecográficas es muy difícil una valoración de todo el contexto anatómico.

Todos estos argumentos han hecho que este tipo de estudios solo se realicen de una forma puntual y que incluso los grupos que más los defendían (7), como el de Gante, los hayan sustituido por el scanner de multidetectores.

\section{ESCANER DE MULTIDETECTORES (MDCT)}

En los últimos 6 ó 7 años la introducción de la Tomografía Axial Computerizada de Multidetectores (Multidetector Row Computed Tomography MDCT) ha significado un tremendo avance en las aplicaciones de la microangiografía computerizada en el estudio de la patología vascular. En el año 2003, tras conocer el uso del MDCT en el estudio de los vasos coronarios, surgió la idea de aplicar toda esta tecnología para el estudio de las perforantes.

El MDCT aporta, como mejora tecnológica respecto a otro tipo de TACs, la capacidad de estudiar gran- 


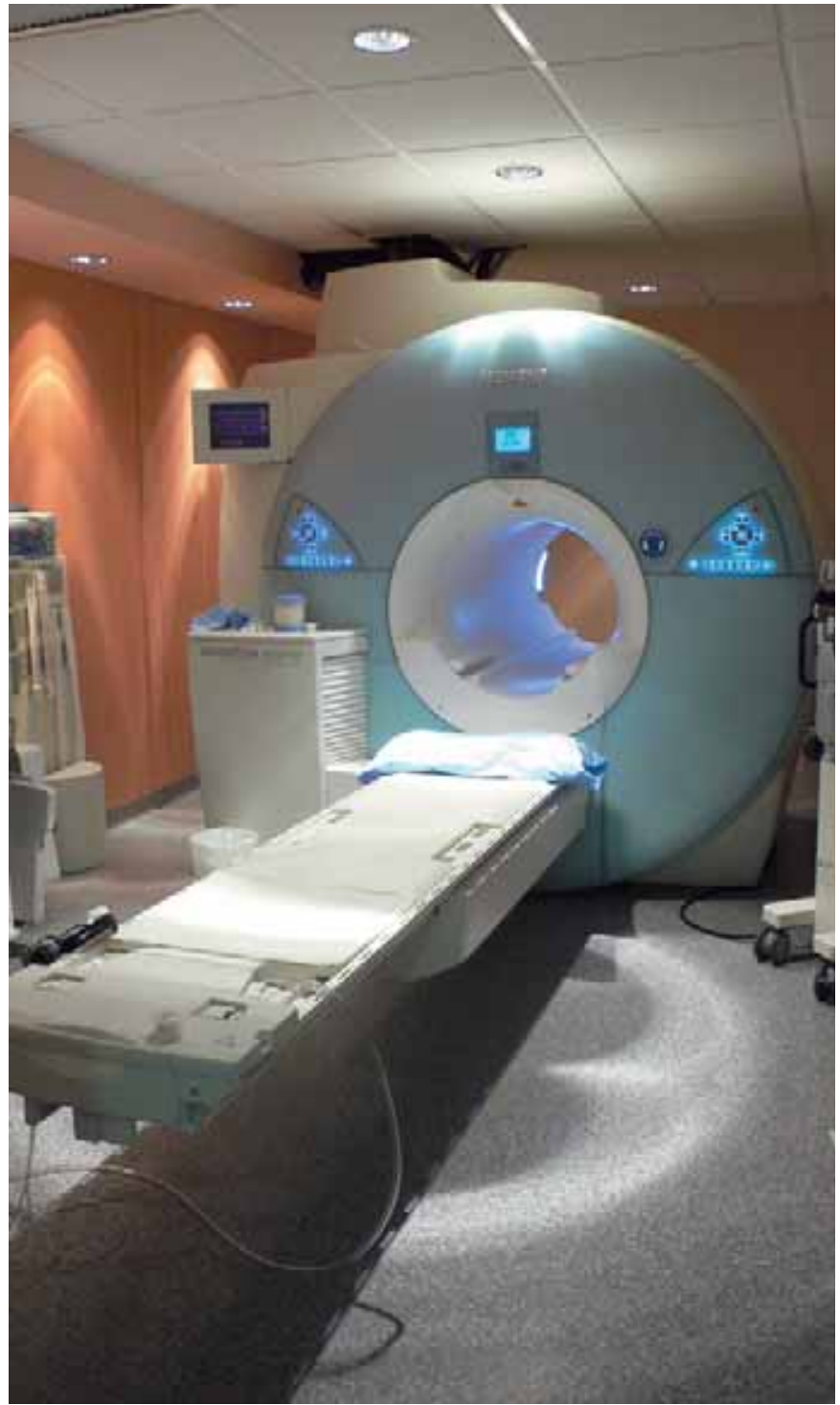

Fig. 5. Escáner de multidetectores Toshiba de 64 coronas.

des extensiones del cuerpo con un tiempo de adquisición mínimo (por ejemplo un tórax y abdomen en 20 segundos) y con una resolución excelente, ya que permite realizar cortes de un grosor menor a $0.5 \mathrm{~mm}$. Todo esto, junto a un índice muy bajo de artefactos en la captación de imagen y un software de reconstrucción tridimensional muy avanzado, hace que la información radiológica que ofrece solo sea comparable con la disección anatómica. Los primeros MDCT que utilizamos eran de 16 coronas; actualmente estamos trabajando con un escáner de 64 coronas, lo que incrementa aún más las posibilidades del estudio (Fig. 5).

En octubre del año 2003 iniciamos un estudio comparativo para poder evaluar la fiabilidad y efectividad del MDCT en el conocimiento de las perforantes abdominales. Una vez finalizado este trabajo, presentamos los resultados en el 8th Internacional Course on Perforator Flaps en Sao Paulo del año 2004.

Actualmente, tras más de 3 años utilizando nuestro protocolo de estudio de perforantes con el MDCT (9),

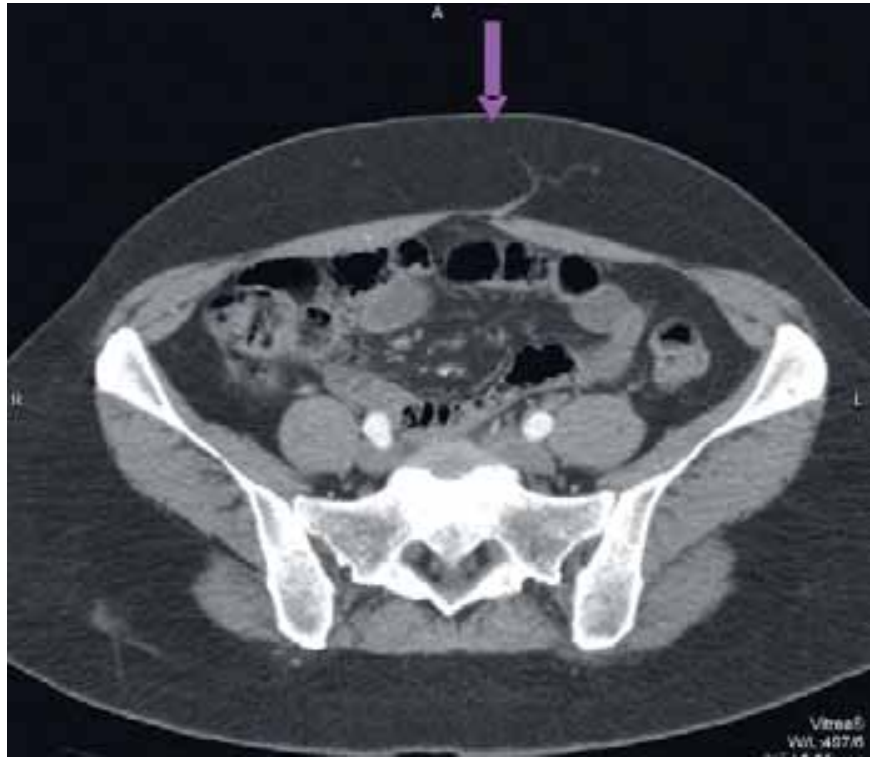

Fig. 6. Visión axial de un MDCT donde podemos ver una perforante medial izquierda.

podemos afirmar que su alta sensibilidad y especificidad, así como un valor predictivo del $100 \%$, lo posicionan como el método de elección para el mapeo de perforantes abdominales y de otras áreas del cuerpo donde la extensión del tejido no permita una rápida visualización intraoperatoria de las perforantes.

Resumiendo el protocolo de estudio que utilizamos (9), podemos decir que lo basamos en el análisis de la información adquirida en un plano axial donde valoramos todas las perforantes y su trayecto desde el vaso origen hasta su localización superficial. Una vez localizadas, se efectúa una doble comprobación desde los planos sagitales y coronales. La exacta localización a nivel cutánea se realiza con una reconstrucción tridimensional y una posterior medición mediante unos ejes de coordenadas virtuales en los que utilizamos una referencia anatómica como centro del eje (en los colgajos abdominales es el ombligo). Las coordenadas exactas de cada perforante son recogidas en una hoja de datos para facilitar su correcto marcaje y dibujo en la piel de los pacientes. Todo el estudio es almacenado en un CD interactivo fácilmente reproducible desde cualquier ordenador (Fig. 6-8).

Las ventajas de esta técnica son:

- Permite una excelente evaluación de la calidad (calibre y flujo), del trayecto y localización de las perforantes en una visión tridimensional.

- El tiempo de realización del MDCT es muy breve. El paciente solo precisa de un tiempo de adquisición dentro del scanner de unos 10 a 20 segundos, lo que se traduce en una mínima incomodidad para el paciente y en la posibilidad de realizar muchas exploraciones en un corto periodo de tiempo. 


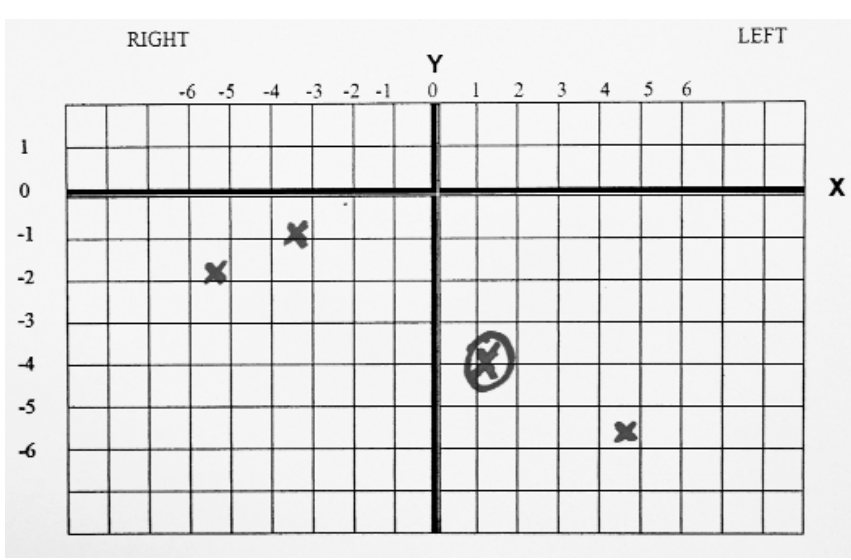

Fig. 7. Hoja de recogida de datos: se ve el eje de coordenadas donde el origen es el ombligo.

- La interpretación por parte del cirujano plástico es muy sencilla, ya que ofrece imágenes anatómicas donde es posible estudiar desde el detalle de la perforante, a una visión global de toda la zona donante.

- Buena reproducibilidad. Toda la información es almacenada en un CD interactivo con un software autoejecutable que permite utilizarlo en cualquier ordenador. Esto nos ofrece la oportunidad de realizar una disección virtual de nuestro paciente antes de la intervención, e incluso si lo creemos conveniente, obtener información en el mismo quirófano.

Como inconvenientes podemos citar la complejidad tecnológica de este tipo de escáner, aunque hoy en día casi todos los grandes centros ya disponen de ellos y la renovación frecuente de este tipo de maquinaria va a hacer que dentro de poco hasta los hospitales pequeños puedan contar con esta tecnología. Otro problema es la radiación a la que sometemos a nuestros pacientes, pero si consideramos que la dosis efectiva de radiación es menor a la de un TAC abdominal convencional y que muchas veces podemos aprovechar un estudio de extensión o de seguimiento que vaya a realizarse para la patología de base por la que vamos a reconstruir al paciente, este punto tampoco debería ser un gran problema en nuestro medio sanitario actual. Por último nombraremos el coste de esta prueba; éste es muy variable de un país a otro, por ejemplo, en los EEUU puede ser de unos 2.000 \$ mientras que en países como Bélgica o Japón es de unos $200 €$. En España, el coste va de los 350 a los $500 €$. Por tanto, si valoramos lo que aporta esta prueba, solo en reducción de tiempo operatorio es más que rentable en el contexto del coste total de la intervención (9).

Después de más de 3 años utilizando este tipo de tecnología para el mapeo de perforantes, sobre todo

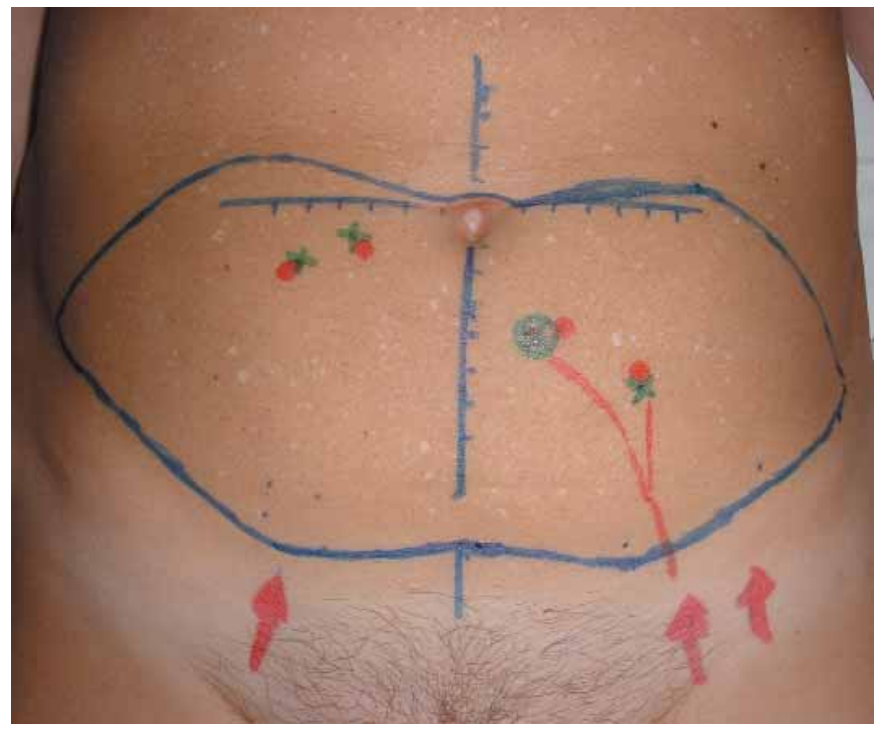

Fig. 8. Diseño preoperatorio de un DIEP con el marcaje de las perforantes a partir de la información obtenida mediante el MDCT.

en colgajos de perforantes abdominales y glúteas, con una casuística de más de 200 pacientes estudiados mediante MDCT, podemos afirmar que para nosotros ha significado una importante reducción del tiempo de disección de las perforantes y una reducción estadísticamente significativa de las complicaciones (10).

\section{Conclusiones}

Con un método fiable de mapeo preoperatorio de perforantes podemos saber cuál es la perforante dominante e idónea par nuestro colgajo y por tanto, podemos realizar un diseño preciso del tejido que necesitamos para nuestro defecto. El límite en las posibilidades reconstructivas vendrá dado por la disponibilidad de las zonas donantes de nuestros pacientes y por la habilidad y capacidad de nuestra técnica quirúrgica.

El diagnóstico y estudio por imagen está sometido a un avance tecnológico tan dinámico, que cualquier tipo de estudio rápidamente se ve mejorado por otro que ofrece nuevas posibilidades. Por tanto, esto nos va a obligar a un esfuerzo e inquietud investigadora continuos que abrirán nuevas fronteras en la cirugía de perforantes.

\section{Dirección del autor}

\section{Dr. Jaume Masià}

Servicio de Cirugía Plástica y Reparadora

Hospital de la Santa Creu i Sant Pau

Sant Antoni M. Claret 167

08025 Barcelona

e-mail: jmasia@santpau.es 


\section{Bibliografía}

1. Taylor GI, Palmer JH.: "The vascular territories (angiosomaes) of the body: experimental study: experimental study and clinical applications". Br J Plast Surg 1987;40:113.

2. Koshima I, Soeda S.: "Inferior epigastric artery skin flaps without rectus abdominis muscle". Br J Plast Surg 1989;42(6):645.

3. Allen RJ, Treece P.: "Deep inferior epigastric perforator flap for breast reconstruction". Ann Plast Surg 1994;32(1):32.

4. Wei, F.C., Jain, V., Celik, N., Chen, H.C., Chuang, D.C., Lin, C.H.: "Have we found an ideal soft-tissue flap? An experience with 672 anterolateral thigh flaps". Plast. Reconstr. Surg. 2002; 09: 2219.

5. Blondeel PN.: "One hundred free DIEP flap breast reconstructions: a personal experience”. Br J Plast Surg 1999 ;52(2):104.
6. Hallock GG.: "Doppler sonography and colour duplex imaging for planning a perforator flap". Clin Plast Surg 2003;30:347.

7. Blondeel PN, Beyens G, Verghaege R, et al.: "Doppler flowmetry in the planning of perforator flaps". Br J Plast Surg 1998;51:202.

8. Voet DVAM, Petrovic M, Masia J, et al.: "Preoperative planning". In: Blondeel PN, Morris SF, Hallock GG, Neligan PC, editors. Perforator flaps. Anatomy, technique and clinical applications. St Louis: Quality Medical Publishing; 2006.

9. Masia J., Clavero J.A., Larrañaga J et al.: "Multidetector-row computed tomography in the planning of abdominal perforator flaps". J Plast Reconstr Aesth Surg 2006; 59:594.

10. Masia J., Calvero JA, Larrañaga J et al.: "Preoperative planning of the abdominal perforator flaps with the multidetector row computed tomography. Three years of experience". Plast. Reconstr. Surg. (In press). 